Rev. Bras. Saúde Prod. Anim., Salvador, v.15, n.2, p.318-326 abr./jun., 2014 http://www.rbspa.ufba.br ISSN 15199940

\title{
Endoparasitismo gastrintestinal em cabras da raça Anglonubiana
}

\author{
Gastrointestinal parasitism in Anglo-Nubiangoats
}

\begin{abstract}
BATISTA, Joilson Ferreira $^{1 *}$; CAMPELO, José Elivalto Guimarães ${ }^{1}$; MORAIS, Marcyele Ferreira $^{2}$; SILVA, Pollyana Oliveira ${ }^{1}$; MAGALHÃES, Pâmela Cristina ${ }^{2}$; BARÇANTE, Felipe Pereira da Silva ${ }^{1}$; MENDONÇA, Ivete Lopes ${ }^{1}$
\end{abstract}

\footnotetext{
${ }^{1}$ Universidade Federal do Piauí, Centro de Ciências Agrárias, Programa de Pós-Graduação em Ciência Animal, Teresina, Piauí, Brasil.

${ }^{2}$ Universidade Federal do Piauí, Centro de Ciências Agrárias, Graduação em Medicina veterinária, Teresina, Piauí, Brasil.

*Endereço para correspondência: joilson.19@ hotmail.com
}

RESUMO

A dificuldade de manutenção do rebanho em condições sanitárias adequada compromete a eficiência da criação de caprinos, principalmente em relação ao controle de endoparasitas. O objetivo com este trabalho foi avaliar a sensibilidade de matrizes da raça Anglonubiana a nematoides gastrintestinais, considerando as cabras estratificadas por estágio fisiológico (lactantes e não lactantes) dentro de três classes de idade. Utilizou-se as características: Ovos por grama de fezes (OPG), Grau de anemia (indicado pelo método Famacha $\left.^{(}\right)$, Peso e Escore da condição corporal e porcentagem de coletas de fezes cujo valor do OPG foi igual a Zero, variável utilizada para demonstrar a resposta ao antihelmintico, disponibilizadas pelo banco de dados dessa raça na UFPI. Os dados são referentes ao período de janeiro de 2009 a dezembro de 2011. As cabras em lactação apresentaram maior valor médio de OPG, maior grau de anemia, menor escore corporal e menor quantidade de coleta de fezes com valor de OPG zero, que as cabras não lactantes, independentemente da classe de idade considerada. Assim, a lactação aumenta a sensibilidade a endoparasitismo em matrizes da raça Anglonubiana. A elevação do OPG tende a se mostrar associada ao maior grau de anemia determinado pelo método Famacha ${ }^{\circledR}$, que levam a redução da condição corporal.

Palavras-chave: caprinos, matrizes, nematódeos, OPG, sensibilidade

\section{SUMMARY}

The difficulty of maintaining adequate sanitary herd health conditions compromises the efficiency of goat breeding, especially in relation to parasite control. The goal with this study was to evaluate the sensibility of female AngloNubiangoats to gastrointestinal nematodes. The does were evaluated stratified by physiological stage (lactating and non-lactating) in three age classes. The following characteristics were used: eggs per gram of feces (EPG), degree of anemia (according to the Famacha ${ }^{\odot}$ method), weight and body condition score and percentage of fecal samples with EPG value equal to Zero, variable used to demonstrate the response to anthelmintic, available in the breed database at UFPI. Data is available for the period from January 2009 to December 2011. Lactating female goats had higher EPG mean value, higher degree of anemia, lower body condition score and fewer feces sampled with zero EPG value, when compared to non-lactating goats, regardless of the age group considered. Therefore, lactation increases sensitivity to endoparasitism in female Anglo-Nubian goats. Elevation of EPG tends to appear associated with an increased degree of anemia determined by the Famacha $($ method, leading to a reduction of body condition.

Keywords: EPG, goats, matrices, nematodes, sensibility 
Rev. Bras. Saúde Prod. Anim., Salvador, v.15, n.2, p.318-326 abr./jun., 2014 http://www.rbspa.ufba.br ISSN 15199940

\section{INTRODUÇÃO}

O rápido retorno econômico da criação de caprino, devido aos seus precoces aspectos produtivos e reprodutivos apresentando ciclo biológico curto, é o principal motivo para sua opção na pecuária (RAMOS et al., 2011). No entanto, a dificuldade de manter $\mathrm{o}$ rebanho em condições sanitárias adequada compromete sua eficiência.

Quanto aos gêneros que infectam caprinos, o Haemonchus é o de maior importância econômica (RIET-CORREA, et al., 2001; AMARANTE, 2005), pois possuem alto grau de hematofagismo e alta capacidade de reprodução, podendo causar várias complicações à saúdo, principalmente dos animais mais susceptíveis.

De forma geral, os animais até a puberdade apresentam grande susceptibilidade à parasitoses, adquirindo com o avançar da idade resistência, porém existem determinadas épocas e condições fisiológicas em que esses animais vão se encontrar mais susceptível (MILLER \& HOROBOV, 2006). Uma dessas condições é (RAHMAN \& COLLINS, 1992), chamada de fenômeno springrise, ou queda da imunidade no peri-parto, que promove o aumento da quantidade de ovos eliminado nas fezes, no entanto os mecanismos pelos quais esse fenômeno ocorre ainda são desconhecidos, porém acredita-se que sejam provocados por imunossupressão de origem endócrina, decorrente de variações hormonais que ocorrem próximas ao parto e durante a lactação (COSTA et al., 2011).

A utilização de antihelmintos no controle de parasitas gastrintestinais em caprinos e ovinos vem sendo analisada, dando espaço para a seleção de animais resistentes aos mesmos e a manipulação da nutrição para aumentar a resistência e/ou resiliência do hospedeiro (TORRES-ACOSTA \& HOSTE, 2008).

$O$ impacto da utilização de animais resistentes na epidemiologia das infecções parasitárias está na diminuição do número de larvas na pastagem, consequentemente reduz a possibilidade de recontaminação dos animais via pastejo (HOSTE \& TORRES-ACOSTA, 2011). Além disso, nos animais considerados "resistentes" os danos causados pelo parasitismo são desprezíveis e nos animais de "resistência intermediária", as infecções sub-clínicas podem causar redução importante na produtividade, enquanto nos animais "susceptíveis" a enfermidade clínica pode se manifestar de forma severa.

O objetivo com este trabalho foi avaliar a sensibilidade de matrizes caprinas a nematoides gastrintestinais, considerando-as estratificadas por estágio fisiológico (lactantes e não lactantes) dentro de idade.

\section{MATERIAL E MÉTODOS}

Nessa pesquisa analisou-se informações coletadas em 80 cabras (média anual) da raça Anglonubiana, no período de janeiro de 2009 a dezembro de 2011, que estão disponíveis no banco de dados do rebanho experimental do Departamento de Zootecnia (CCA/UFPI), localizado em Teresina (latitude de $5^{\circ}$ 5'20" sul e longitude de $42^{\circ} 48^{\prime} 07^{\prime \prime}$ oeste). O banco de dados é formado com um histórico completo dos animais, desde o nascimento até o óbito, com informações reprodutivas, sanitárias e produtiva dos animais. $\mathrm{O}$ tratamento com antihelmíntico no rebanho seguiu o estágio fisiológico das matrizes, com aplicações feitas da seguinte forma: a $1^{a}$ aplicação na desmama (120 dias pós o parto), a $2^{\mathrm{a}}$ no terço final da gestação, a $3^{a}$ após o parto, a $4^{\mathrm{a}}$ em torno de 60 dias do parto e finalizou-se o ciclo com a $5^{\mathrm{a}}$ 
vermifugação na desmama, que correspondeu à primeira do ciclo seguinte de produção do animal. As características utilizadas para identificar o perfil de sensibilidade dos animais a nematoides gastrintestinais foram: Ovos por grama de fezes (OPG), Grau de anemia pelo método Famacha $^{\odot}$, Peso, Escore da condição corporal e Porcentagem de coletas de fezes com valor do OPG igual a Zero, essa característica foi estudada com a finalidade de observar a resposta individual dos animais ao antihelmintico. Todas as variáveis foram coletadas no mesmo dia em que as coletas de fezes foram realizadas.

A coleta de fezes ocorreu a cada 40 dias (nove coleta animal/ano), sempre antes da utilização de antihelmintico nos animal, com a retirada das fezes diretamente da ampola retal, acondicionada em saco plástico individual para análise de $\mathrm{OPG}$ no Laboratório de Sanidade Animal CCA/UFPI, realizada com a técnica de Mc Master modificada por Whitlock e coprocultura para identificação do gênero infectante, segundo Ueno \& Gonçalves (1998).

O Escore da condição corporal do animal foi obtido com atribuição de notas de 1 a 5 (sendo 1 animal muito magro e 5 obeso) por três avaliadores distintos e considerado a média (MACHADO et al., 2008). O grau de anemia do animal pelo método Famacha $^{\circledR}$, foi avaliado com a atribuição de nota de 1 a 5 à tonalidade da conjuntiva dos animais por três avaliadores, correspondendo do vermelho-rosado ao branco pálido, respectivamente, utilizando cartão guia desenvolvido para utilização a campo (MOLENTO, 2004).

$\mathrm{Na}$ edição dos dados, realizada em Planilha eletrônica, os animais foram estratificados em três classes de idade: "cabra nova" (com menos de três anos); "cabra de idade intermediária" (com idade entre três e seis anos) e "cabra velha" (com mais de seis anos). Em cada classe estratificou-se os animais em cabras "não-lactantes" e "lactantes" (excluindo-se informações de cabras no terço final de gestação, resultando em número desigual de animais por estágio fisiológico) resultando em dados de 105 matrizes lactantes e 79 não lactantes ao longo dos 3 anos. Em seguida calculouse a porcentagem de coletas de fezes com valor do OPG igual a zero, bem como o percentual de animais com valor de OPG zero em mais de uma coleta no mesmo estágio. Os dados foram submetidos a análises utilizando-se estatística descritiva, com determinação de média e desvio-padrão.

\section{RESULTADOS E DISCUSSÃO}

Observa-se na Tabela 1 que a faixa etária dos animais avaliados ficou entre 2,2 anos (cabras novas) e 7,2 anos (cabras velhas). Em cada classe de idade a quantidade de cabras nãolactante foi menor que aquelas que se encontravam em lactação. Nessa criação os animais são divididos em dois grupos durante as estações de monta, com cada uma em um semestre do ano, isso fez com que no período das coletas uma parte das matrizes estivessem lactantes, outra parte gestante e uma menor proporção vazia (apenas animais que não gestaram em nenhuma das estações de monta). Levando as cabras com a gestação confirmada, ficarem não contemporânea em termos de estágio fisiológico com as demais e as coletas nestas foram desconsideradas. 
Rev. Bras. Saúde Prod. Anim., Salvador, v.15, n.2, p.318-326 abr./jun., 2014 http://www.rbspa.ufba.br ISSN 15199940

Tabela 1. Médias e desvios-padrão de Ovo por grama de fezes (OPG), Grau de anemia Famacha $^{\odot}$, Peso e Escore Corporal de cabras da raça Anglonubiana, estratificadas por estágio fisiológico e idade

\begin{tabular}{llcccc}
\hline Idade em anos & $\begin{array}{c}\text { Estágio } \\
\text { Fisiológico }\end{array}$ & OPG & $\begin{array}{c}\text { Grau de anemia } \\
\text { (nota 1 a 5) }\end{array}$ & Peso (kg) & $\begin{array}{c}\text { Escore } \\
\text { (nota 1 a 5) }\end{array}$ \\
\hline $2,2 \pm 0,4$ & Não lactante & $500 \pm 1060$ & $2,5 \pm 0,66$ & $35,3 \pm 5,3$ & $2,60 \pm 0,42$ \\
(novas) & Lactante & $1916 \pm 2793$ & $3,1 \pm 0,72$ & $34,4 \pm 5,3$ & $2,01 \pm 0,43$ \\
$4,7 \pm 0,9$ & Não lactante & $946 \pm 3229$ & $3,0 \pm 0,54$ & $43,6 \pm 5,9$ & $2,61 \pm 0,54$ \\
(intermediária) & Lactante & $1490 \pm 3160$ & $3,2 \pm 0,63$ & $40,7 \pm 4,6$ & $2,23 \pm 0,90$ \\
$7,2 \pm 1,0$ & Não lactante & $746 \pm 1394$ & $3,2 \pm 0,65$ & $45,0 \pm 5,6$ & $2,30 \pm 0,59$ \\
(velhas) & Lactante & $1957 \pm 3458$ & $3,4 \pm 0,72$ & $43,0 \pm 6,0$ & $2,03 \pm 0,45$ \\
\hline
\end{tabular}

A maior sensibilidade, representada pelo maior resultado de OPG, das cabras em lactação diante da agressão dos endoparasitas foi verificada nas três classes de idade. Constatou-se que o valor médio do OPG das cabras em lactação superou o dos animais não lactantes e com tendência crescente com o envelhecimento do animal, concordando com afirmações de Houdijk (2008) que a lactação predispõe mais a cabra a infecção parasitária. $\mathrm{O}$ fenômeno do peri-parto, que vai do terço final da gestação a lactação, pode ser devido a baixa imunidade dos animais, visto que nesse período os animais apresentam imunossupressão (CIARLINI et al., 2000) e (PINTO et al., 2008). Segundo Gennari et al. (2002) o aumento do valor do OPG apresenta relação direta com a queda da imunidade, o que permite o desenvolvimento de larvas que se encontram em hipobiose, além do estabelecimento de novas, ou favorece o aumento da fecundidade de parasitas adultos existentes, que resulta em aumento no número de ovos eliminados nas fezes (STEAR et al., 1997).

O elevado valor do desvio-padrão, independentemente da idade do animal e do estágio fisiológico, indicou distribuição agregada dessa característica no rebanho, típica das infecções parasitárias, onde poucos animais suscetíveis são os grandes responsáveis pela contaminação do rebanho, como constatado por Gasbarre et al. (2001), ao estudar o papel do sistema imunitário e do genoma da resistência a nematoides gastrintestinais em bovinos. A maior sensibilidade das cabras em lactação diante da ação dos endoparasitas também foi detectada pelo grau de anemia dos animais, medido pelo método $\mathrm{Famacha}^{\circledR}$, pois as cabras lactantes apresentaram sempre maiores valores, numa indicação de relação direta com o comportamento do OPG. Foi observado também que o grau de anemia apresentou-se crescente com o avanço da idade do animal, independentemente das cabras velhas estarem lactantes ou não. Nesse caso convém salientar que o valor médio do OPG foi igual a 746, resultados que indicam exposição continua das cabras a infecção por nematódeos gastrintestinais. Quanto a presença de parasitas, o gênero Haemonchus foi o de maior frequência e permanecendo distribuído ao longo dos anos neste rebanho, quase sempre apresentando altos percentuais (Figura 1), o que explica o elevado grau de anemia dos animais. $O$ fato do Haemonchus causar grandes perdas econômicas está associado ao seu alto grau de hematofagismo e da capacidade de inoculação de substância 
anticoagulante no local onde se fixam, além da sua alta capacidade de reprodução. Esses resultados também foram corrobora com os de Costa Júnior et al. (2005) e Silva (2011), que constataram o gênero Haemonchus distribuído continuamente ao longo dos meses estudados, mantendo-se em maiores níveis durante a estação úmida.



Figura 1. Percentagem dos gêneros de nematoides gastrintestinais encontrados no período de 2009 a 2011 no rebanho da UFPI, em Teresina

Assim como em muitos estudos de parasitose o gênero Trichostrongylos foi o que apareceu em segundo lugar (ARAÚJO et al., 2007; COSTA et al., 2011). Diversas estratégias têm sido estudadas no sentido de promover métodos que auxiliem no controle dos nematóides gastrintestinais de pequenos ruminantes. Desta forma, o Controle Integrado de Parasitos (CIP) que é a adoção combinada de métodos de utilização de antihelmínticos com métodos que não utilizem estes produtos, dentre eles pode-se citar: seleção de animais resistentes, diminuição de larvas infectantes na pastagem e instalações, vacinas, fitoterapia e método Famacha ${ }^{\circledR}$. A finalidade destas estratégias é manter níveis aceitáveis de infecção em animais de produção e diminuir a utilização de antihelmínticos, essa estratégia vem sendo cada vez mais difundida (CAVELE, 2009). O CIP está aos poucos sendo implantado na criação, com a limpeza das instalações, rodizio de pastagens, uso do método Famacha ${ }^{(}$ e utilização de antihelmintico, com teste de resistência ao mesmo anualmente. Dessa forma e com a inexistência atualmente de vacinas disponíveis no mercado, recomenda-se iniciar seleção de animais resistentes nesse rebanho.

$\mathrm{O}$ peso corporal médio das cabras aumentou com a idade nos dois estágios fisiológicos avaliados, apresentando-se entre 34 a $45 \mathrm{~kg}$. Contudo o peso desses animais está relacionado ao tamanho corpóreo desse, demonstrando um indicativo de crescimento dos animais com o avanço da idade. Já a condição corporal da cabra tendeu a reduzir, numa indicação de diminuição da capacidade de mantença com o aumento da idade no rebanho, de forma mais intensa na lactação. 
A maior suscetibilidade das cabras para enfrentar parasitoses durante a lactação também ficou bem evidente ao se analisar o percentual de coletas de fezes cujo valor do OPG foi zero. Constatouse que, independentemente da idade considerada, nas cabras não lactantes foi maior o percentual de coletas com o valor do OPG igual a zero, sendo essa diferença mais pronunciada nas cabras novas $(32,61$ contra $12,86 \%)$ e nas velhas $(26,6$ contra $12,7 \%)$ (Tabela 2). A quantidade de cabras que apresentou mais de uma coleta de fezes com valor de OPG igual a zero também apresentou esse mesmo comportamento, sendo verificado nas cabras velhas não lactantes, que $37,5 \%$ delas apresentaram mais de uma coleta zeradas.

Tabela 2. Quantidade de cabras (n), cabras com coletas de OPG zero (\%), média e desvio-padrão do número de coleta de fezes por cabra e número de coletas de fezes com OPG zero (\%), em fêmeas Anglonubiana estratificadas por estágio fisiológico dentro de idade

\begin{tabular}{llcccc}
\hline Idade em anos & $\begin{array}{c}\text { Estágio } \\
\text { Fisiológico }\end{array}$ & $\begin{array}{c}\mathrm{N}^{\mathbf{o}} \text { de cabras } \\
(\mathrm{n})\end{array}$ & $\begin{array}{c}\text { Cabras com } \\
\text { OPG Zero(\%) }\end{array}$ & $\begin{array}{c}\mathrm{N}^{\mathbf{0}} \text { de Coleta } \\
\text { por cabra }\end{array}$ & $\begin{array}{c}\text { Coletas com } \\
\text { OPG Zero(\%) }\end{array}$ \\
\hline $2,2 \pm 0,4$ & Não lactante & 17 & 25,0 & $3,6 \pm 0,7$ & 32,61 \\
(novas) & Lactante & 24 & 11,1 & $4,6 \pm 0,6$ & 12,86 \\
$4,7 \pm 0,9$ & Não lactante & 38 & 36,8 & $3,4 \pm 0,5$ & 26,72 \\
(intermediária) & Lactante & 49 & 18,4 & $5,7 \pm 0,7$ & 19,70 \\
$7,2 \pm 1,0$ & Não lactante & 24 & 37,5 & $3,4 \pm 0,6$ & 26,60 \\
(velhas) & Lactante & 32 & 27,3 & $5,8 \pm 0,8$ & 12,70 \\
\hline
\end{tabular}

${ }^{1}$ Porcentagem de cabras que apresentaram coleta com valor de OPG Zero.

A importância desse resultado está no fato de indicar que o esquema de utilização de antihelmintico usado no rebanho, pode está apresentando eficiência diferenciada em cada classe de idade. Por outro lado, pode indicar que não foi eficiente para atingir os parasitas nos animais, visto que $2 / 3$ continuaram contaminados, consequentemente, com potencial para reinfestar os pastos, que por sua vez contribuíram para infectar novamente os animais, nos meses que o sistema de rotação de pastejo não foi eficiente. Além disso, a alta exposição dos animais aos antihelmintos pode reduzir o tempo de eficiência desses medicamentos no combate aos parasitas, visto que, há uma grande tendência de aumentar os níveis de resistência pela persistência de utilização dessas drogas (SCZESNYMORAES et al., 2010).

A presença de animais com valor de OPG igual a zero em rebanhos onde a média de OPG é alta também pode ser devido a variação genética de cada indivíduo. Nunes et al. (2007) estudando a variabilidade genética de resistência a nematódeos gastrointestinais em ovinos através de marcadores moleculares observou que existe variabilidade genética da característica resistência a endoparasitos dentro de rebanhos.

Quanto aos gêneros de nematódeos gastrointestinais presente no rebanho durante o estudo, observou-se que $\mathrm{o}$ Haemonchus e o Trichostrongylus foram detectados em todas as análises, variando de mais de $80 \%$ de predominância do 
gênero Trichostrongylus no mês de dezembro de 2009 a quase $100 \%$ de predominância do gênero Haemonchus em janeiro de 2011. Essa variação dos gêneros predominantes pode ser devido a maior sensibilidade individual de cada gênero a determinados princípio ativo de drogas antiparasitárias, visto que, no rebanho estudado adota-se a prática de rotação de princípio ativo no controle de parasitas gastrointestinais.

Os gêneros Oesophagostomum e Coopéria apareceram em quantidades expressivas em janeiro de 2010. Nos outros meses estudados apareceram em pequenas quantidades e muitas vezes não foram detectados.

Dos quatro gêneros encontrados na cultura da matéria fecal no rebanho, o Haemonchus foi o de maior prevalência com média de 56\%, seguido do Trichostrongylus com 38\%, Oesophagostomum 4\% e Cooperia $2 \%$. A lactação aumenta a suscetibilidade a endoparasitismo em matrizes da raça Anglonubiana, de forma mais intensa nas cabras novas e nas mais velhas.

A elevação do OPG tende a se mostrar associada ao maior grau de anemia, que levam a redução da condição corporal.

\section{REFERÊNCIAS}

AMARANTE, A.F.T. Controle de verminose ovina. Revista do Conselho Federal de Medicina Veterinária,v.11, n.34, p.19-30, 2005.

ARAÚJO, J.V.; RODRIGUES, M.L.A.; SILVA, W.W.; VIEIRA, L.S. Controle biológico de nematóides gastrintestinais de caprinos em clima semi-árido pelo fungo Monacrosporium thaumasium. Pesquisa Agropecuária Brasileira, v.42, n.8, p.1177-1181, 2007.
CAVELE, A. Variáveis clínica, parasitológica, hematológica e Bioquímica de caprinos e ovinos infectados naturalmente Por nematóides gastrintestinais sob o mesmo sistema de produção. 2009. 99f. Dissertação (Mestrado em Ciência Animal nos Trópicos) - Universidade Federal da Bahia, Salvador.

CIARLINI, P.C.; MOTTA, T.; KOHAYAGAWA, A.; ALENCAR, N.X.; AMARANTE, A.F.T.; LOPES, R.S.; LENZ, F.F. Serum persinogen concentration in suffolk and polwarth ewes at the end of gestation, during lactation and after weaning. Revista Brasileira de Parasitologia Veterinária, v.9, n.1, p.17-21, 2000.

COSTA JÚNIOR, G.S.; MENDONÇA, I.L.; CAMPELO, J.E.G.;

CAVALCANTE, R.R.; FILHO, L.A.D.; NASCIMENTO, I.M.R.; ALMEIDA, E.C.S.; CHAVES, R.M. Efeito de vermifugação estratégica, com princípio ativo à base de ivermectina na incidência de parasitos gastrintestinais no rebanho caprino da UFPI. Ciência Animal Brasileira, v.6, n.4, p.279-286, 2005.

COSTA, V.M.M.; SIMÕES, S.V.D.; RIET-CORREA, F. Controle das parasitoses gastrintestinais em ovinos e caprinos na região semiárida do Nordeste do Brasil. Pesquisa Veterinária Brasileira, v.31, n.1, p.6571, 2011.

GASBARRE, L.C; LEIGHTON, E.A.; SONSTEGARD, T. Role of bovine immune system and genome in resistance to gastrointestinal nematodes. Veterinary Parasitology, v.98, p.5164. 2001. 
Rev. Bras. Saúde Prod. Anim., Salvador, v.15, n.2, p.318-326 abr./jun., 2014 http://www.rbspa.ufba.br ISSN 15199940

GENNARI, S.M.; BLASQUES, L.S.; RODRIGUES, A.A.R.; CILENTO, M.C.; SOUZA, S.L.P.; FERREIRA, F. Determinação da contagem de ovos de nematódeos no período peri-parto em vacas. Brazilian Journal of Veterinary Research and Animal Science, v.39, n.1, p.32-37, 2002.

HOSTE, H.; TORRES-ACOSTA, J.F.J. Non chemical control of helminthes in ruminants: Adapting solutions for changing worms in a changing world. Veterinary Parasitology, v.180, p.144-154, 2011.

HOUDIJK, J.G.M. Influence of periparturient nutritional demand on resistance to parasites in livestock.

Parasite Immunology, v.30, p.113121, 2008.

MACHADO, R.; CORREAA, R.F.; BARBOSA, R.T.; BERGAMASCHI, M.A.C.M. Escore da condição corporal e sua aplicação no manejo reprodutivo de ruminantes. São Carlos, SP: Embrapa Pecuária Sudeste, 2008. 16p. (Circular Técnica, 57).

MILLER, J.E.; HOROHOV, D.W. Imunological aspects of nematode parasite control in sheep. Journal of Animal Science, v.84, p.124-132, 2006.

MOLENTO, M.B. Resistência de helmintos em ovinos e caprinos. Revista Brasileira de Parasitologia Veterinária, v.13, p.82-85, 2004.

NUNES, A.P.; OLIVEIRA, A.C.; BERN, M.E.A.; BORBA, M.F.S.; ECHEVARRIA, F.; VAZ, C.M.; CARVALHO, F.I.F. Estudo da variabilidade genética de resistência a nematódeos gastrintestinais em ovinos da raça corriedale com marcadores
RAPD. Revista Brasileira de

Agrociência, v.13, n.1, p.25-33, 2007.

PINTO, J.M.S.; OLIVEIRA, M.A.L.;

ÁLVARES, C.T.; DIAS, R.C.;

SANTOS, M.H. Relação entre o periparto e a eliminação de ovos de nematóides gastrintestinais em cabras Anglo Nubiana naturalmente infectadas. Revista Brasileira de Parasitologia Veterinária, v.17, p.138-143, 2008. Supl.1.

RAHMAN, W.A.; COLLINS, G.H. An association of faecal egg counts and prolactin concentrations in sera of parturient angora goats. Veterinary Parasitology, v.43, n.1-2, p.85-91, 1992.

RAMOS, A.F.; ALBUQUERQUE, M.S.M.; MARIANTE, A.S. Banco Brasileiro de Germoplasma Animal: desafios e perspectivas da conservação de caprinos no Brasil. Revista Brasileira de Reprodução Animal, v.35, n.2, p.104-107, 2011.

RIET-CORREA, F.; SCHILD, A.L.; MÉNDEZ, M.C.; LEMOS, R.A.A. Doenças de ruminantes e equinos. 2.ed. São Paulo: Varela, 2001. 574p.

SCZESNY-MORAES, E.A.; BIANCHIN, I.; SILVA, K.F.; CATTO, J.B.; HONER, M.R.; PAIVA, F. Resistência antihelmíntica de nematóides gastrintestinais em ovinos, Mato Grosso do Sul. Pesquisa Veterinária Brasileira, v.30, n.3, p.229-236, 2010.

SILVA, N.C.S. Efeitos ambientais que interferem no endoparasitismo em matrizes da raça Anglonubiana em Teresina - Piauí. 2011. 62p.

Dissertação (Mestrado em Ciência Animal) - Universidade Federal do Piauí, Teresina. 
Rev. Bras. Saúde Prod. Anim., Salvador, v.15, n.2, p.318-326 abr./jun., 2014 http://www.rbspa.ufba.br ISSN 15199940

STEAR, M.J; BAIRDEN, K.; BISHOP, S.C.; BUITKAMP, J.; DUNCAN, J.L.; GETTINBY, G.; MCKELLAR, Q.A.; PARK, M.; PARKINS, J.J.; REID,

S.W.; STRAIN, S.; MURRA,Y.M. The genetic basis of resistance to Ostertagia circumcincta in lambs. Veterinary Journal, v.154, p.111-119, 1997.

TORRES-ACOSTA, J.F.J.; HOSTE, H. Alternative or improved methods to limit gastro-intestinal parasitism in grazing sheep and goats. Small

Ruminant Research, v.77, n.2, p.159173, 2008.
UENO, H.; GONÇALVES, P.C. Diagnostico das helmintoses de ruminantes. 4.ed. Tokyo: Japan International Cooperationagency, 1998. p.14-16.

Data de recebimento: 19/09/2013

Data de aprovação: 16/05/2014 